\title{
Illustration-inspired techniques for visualizing time-varying data
}

\author{
Alark Joshi* \\ University of Maryland Baltimore County
}

\begin{abstract}
Traditionally, time-varying data has been visualized using snapshots of the individual time steps or an animation of the snapshots shown in a sequential manner. For larger datasets with many timevarying features, animation can be limited in its use, as an observer can only track a limited number of features over the last few frames. Visually inspecting each snapshot is not practical either for a large number of time-steps.

We propose new techniques inspired from the illustration literature to convey change over time more effectively in a time-varying dataset. Speedlines are used extensively by cartoonists to convey motion, speed, or change over different panels. Flow ribbons are another technique used by cartoonists to depict motion in a single frame. Strobe silhouettes are used to depict previous positions of an object to convey the previous positions of the object to the user. These illustration-inspired techniques can be used in conjunction with animation to convey change over time.
\end{abstract}

Keywords: Flow visualization, Non-photorealistic rendering, time-varying data, illustration

\section{INTRODUCTION}

Visualization of time-varying data has been a challenging problem due to the nature and the size of the datasets. The naïve approach to visualizing time-varying data is to render the three-dimensional volume at each individual time step using standard volume rendering techniques [2]. This technique relies heavily on the user's ability to identify and track regions of interest over time. At the same time, the number of snapshots generated can be quite high (100-3000), requiring considerable effort for the user to track features. To reduce this effort, we draw inspiration from the illustration literature to enable us to convey change over time more succinctly. Time-varying data are three-dimensional snapshots of a process captured at regular time intervals. Domains such as computational fluid dynamics, weather forecasting and medical scans (ultrasound) generate timevarying data.

Time-varying data visualization consists of three steps. First, the dataset must be analyzed to identify interesting features. Features are regions of interest depending on the scientific domain. Feature extraction can be done manually where a user selects features, semi-automatically (where an algorithm identifies features which are validated by a user), or automatically identifying features by analyzing different time steps. Attributes are identified for each feature to quantify it [17].

The second step in visualizing these datasets is feature tracking. The extracted features (from different time steps) are tracked over the time steps. Feature tracking requires the ability to identify the features and correlate them over time.

The third step is visualizing this tracked information along with the actual time-varying data. The visualization conveys the change

*e-mail: alark1@cs.umbc.edu

†e-mail:rheingan@cs.umbc.edu

IEEE Visualization 2005

October 23-28, Minneapolis, MN, USA

0-7803-9462-3/05/\$20.00 @2005 IEEE.

\author{
Penny Rheingans ${ }^{\dagger}$ \\ University of Maryland Baltimore County
}

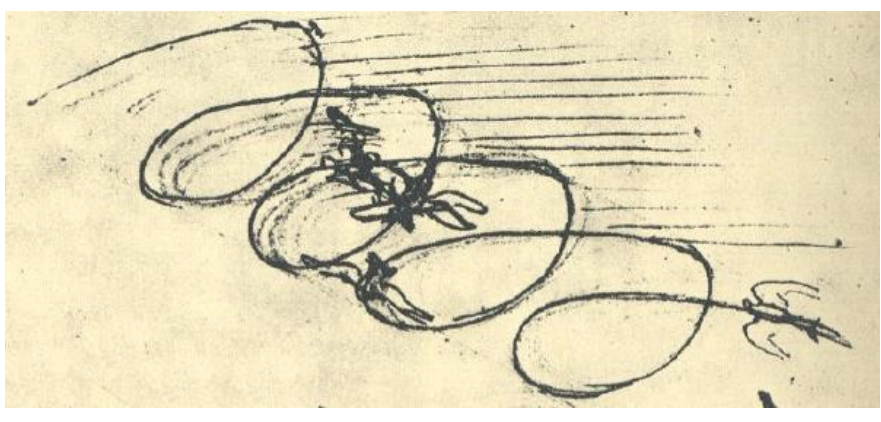

Figure 1: This illustration depicts the motion of a bird in flight with the abstract path traversed by the bird and intermediate position indications [13].

over time in the underlying data. The feature extraction and feature tracking approach is fairly common in time-varying dataset visualization [20].

Generally, identifying and visualizing features over time is a particularly hard task, even for the well-trained eye. The problems are numerous ranging from the large number of time steps that are rendered to the ability of the user/viewer to identify and visually track a particular feature of interest. There is always the problem of occlusion of the feature of interest by another uninteresting feature. The problem would be further complicated by issues such as the large number of features in each time step.

In an experiment by Pylyshyn [15], it was found that observers can track a maximum of five independently moving objects at the same time. As the speed of the moving objects and the number of objects increases, the performance of the observer dropped considerably. The results of this experiment are particularly significant because they serve as a motivation for our problem of effectively depicting change over time for datasets containing many features. It is not uncommon to have 10-20 features in a particular dataset and the experimental results above clearly state that is impossible for the human visual system to track their paths over time.

Illustrations have been used extensively to convey information not easily conveyed by photographs [7]. Illustrations are able to convey information easily, drawing the viewer's attention to the important details and abstracting out the irrelevant details. Illustrators have used numerous techniques to depict change over time in a single image [14].

Leonardo Da Vinci in his treatise on the the flight of birds [13] writes "The lines of the movements made by birds as they rise are of two kinds, one which is always spiral in the manner of a screw, and the other is rectilinear and curved. That bird will rise up to a height which by means of a circular movement in the shape of a screw makes its reflex movement against the coming of the wind and against the flight of this wind, turning always upon its right or left side." To illustrate this effect, he drew the illustration reproduced in Figure 1 that depicts the motion of the bird in flight. The illustration conveys the motion to the viewer by using lines to approximate the path taken by the bird to ascend into the sky. The lines do not connect exact positions of the bird as it took flight, but is an abstraction of those positions. Illustrators tend to rely more on 


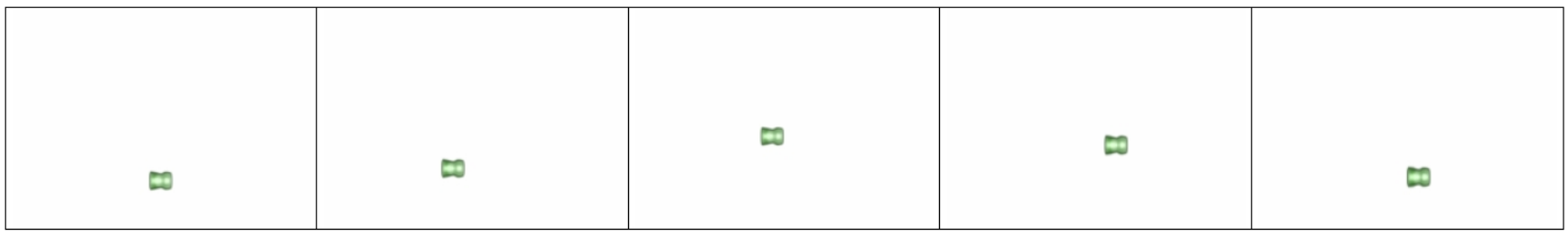

Figure 2: This image depicts the motion of a feature in an experimental data set over time. The direction of motion of this feature is not clear from these snapshots.

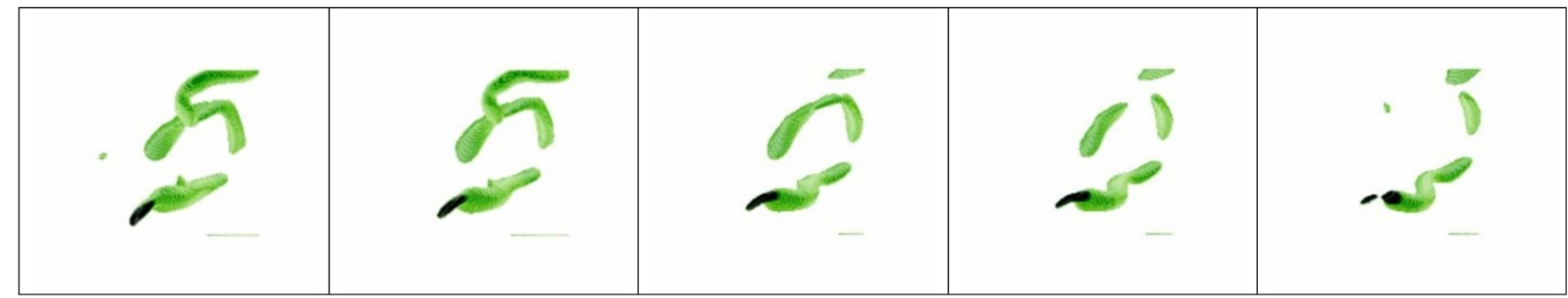

Figure 3: These are a set of snapshots from successive time steps of a volume. As is evident from looking at the snapshots, it is very hard to correlate and track a particular feature over different time steps. The problem partly lies in the fact that many features are very short lived which is why we used the coefficient of variation measure to identify stable, interesting features (illustration features).

abstraction than accuracy when conveying change over time.

The problem of efficiently and succinctly displaying a minimal set of images that conveys information about the interactions within a time-varying dataset is still unsolved. We propose the use of illustration-inspired NPR techniques to convey change in the volumetric data over time.

We have identified a few techniques from the illustration literature and apply them to depict change over time in time-varying datasets. Speedlines are one such technique which follows features and draws the user's attention to regions of interest. A group of speedlines together form flow ribbons that convey change of a feature over time more succinctly. Researchers in the field of visualization have used opacity-based techniques to draw the user's attention to a particular feature in a visualization [20]. We extend this technique to vary the opacity of features as they transform over time. We found that opacity-based techniques when coupled with speedlines are a much stronger indicator of change over time than just using opacity-based techniques.

\section{RELATED WORK}

Research in the field of visualizing time-varying datasets has been focused around the data to be visualized. Computational fluid dynamics (CFD) simulations led part of the early research in the field of visualizing time varying data. Samtaney et al. [17] were the first to identify techniques in the field of computer vision and extend them from the two-dimensional image domain to the threedimensional volume domain. Feature extraction techniques and feature attributes were identified. Feature tracking was introduced in this paper and was later improved by Silver et al. [20].

Time-varying data visualization currently focuses on using compression [18] and optimized data structures and algorithms [22, 24] to manage data efficiently. Recent advances in graphics hardware have also been leveraged to interactively visualize time-varying data [12].

At the same time, conveying information to the viewer using non-traditional techniques is crucial. As the datasets get bigger, using smart techniques to convey the information contained within the dataset becomes more important. Non-photorealistic rendering (NPR) has shown great promise in conveying information. Many researchers have used such NPR techniques for visualizing medical and scientific datasets $[16,10,11,3,6]$. Ma et al. [21] have applied non-photorealistic rendering techniques to time varying data visualization. They used techniques such as gradient, silhouette and depth enhancement to provide more spatial and temporal cues. Svakhine et al. [23] have extended volume illustration techniques [16] for time-varying data and have applied Schlieren and shadowgraphy techniques from the field of photography to convey change over time.

\section{Approach}

Our approach to time-varying data visualization is inspired by the illustration literature. We first preprocess the time steps to analyze them and identify features of interest. In the second step, the identified features are correlated between each time step to facilitate tracking. In order to track a feature, we calculate the centroid of the feature and track it over time. Since the centroid of a feature can fall outside the feature, we also track the extreme points of the volume at each time step to provide us with more information for tracking. The centroid and the extreme points are used by our techniques to convey the direction of motion of the feature over time.

\subsection{Feature identification}

We have used feature-extracted data from the Rutgers data repository. Every voxel in each time step has an identifier that indicates the flow feature at every time step, making it possible to track a particular set of flow features.

In this process of enhancing the rendering to draw the user's attention to regions of interest, we first need to identify features of interest, which we shall call illustration features to avoid confusion.

In the preprocessing step, we analyze the time-varying data set to identify flow features that are most actively moving (unstable), mostly stable as well as significantly larger compared to its surrounding features. This preprocessing allows us to target a certain 
class of flow features (stable/unstable) that facilitiates more effective visualizations.

We define a quantity called the temporal variation. Temporal variation of a feature is defined as a quantity that measures the amount of change that a feature undergoes over time. We used the coefficient of variation (COV), a statistical measure of the standard deviation of a variable. The Coefficient of variation has been used for transfer function generation in time-varying data [8], for accelerating volume animation [19] and for accelerating the rendering of time-varying data using TSP trees [18, 4].

The COV for a data value is given by:

$$
c_{v}=\frac{\sigma_{v}}{\overline{o_{v}}}
$$

In the above equation, $\overline{o_{v}}$ is the mean of the sample $o_{v, t}$ under consideration over $n$ time steps. $\sigma_{v}$ is the standard deviation of $o_{v, t}$ from its calculated mean. The $\mathrm{COV} c_{v}$ is calculated by dividing the deviation $\sigma_{v}$ by the overall mean $\overline{o_{v}}$. A larger COV implies high variation and less stability and similarly a smaller value implies more stability over time, implying that it may be a feature of interest (illustration feature).

Temporal variation is computed by comparing consecutive timesteps. For each pair of consecutive time steps, we compute a gradient volume that contains temporal gradients for each voxel. The temporal COV is then computed for the temporal gradients. Temporal gradients give a sense of how the voxel density changed over time for a particular voxel.

We use an experimental dataset to convey the efficacy of the techniques and then present our results on actual CFD data. In Figure 2 , the feature is moving in a circular fashion, but it is not at all apparent from visualizing the individual time steps as shown in the figure. Figure 3 shows the snapshots for five consecutive timesteps in a time-varying dataset. It is evident from these figures that tracking a particular feature over time is hard.

\subsection{Speedlines}

Speedlines can be defined as lines that convey information to the user about the path traversed by a particular feature over time. They are basically lines that follow a particular feature over time. Illustrators have used speedlines to convey motion by altering the characteristics of these lines. The thickness, the line-style and variation of the opacity are the types of characteristics that successfully convey the change.

For example, in Figure 4 the illustrator has been successful in conveying the motion of the pitcher's arm to the viewer [9]. In particular, the thickness of the lines is varied to show the direction of motion of the pitcher's hand. It is also important to note that the curve tracing the pitcher's arm is smooth and not irregular. It is an abstraction of the actual movement of a pitcher's arm. The motion of the baseball towards the viewer is successfully depicted by the speedlines that start out thin to depict the origin of motion, but later thicken to imply the increased intensity of force at that point and then again start thinning towards the end.

Illustrators use thicker, denser lines to represent older time instants and lighter, thinner lines to represent newer time steps. Figure 5 shows an illustration with speedlines. The older lines are thicker and darker and they get thinner and lighter as they approach the man.

In Figure 6 we have identified one feature and conveyed its motion over twelve timesteps. To convey the notion of time, we have used opacity based speedlines. The darker, thicker line depicts an older time step whereas the lighter, thinner regions of the speedline depict a more recent time step. By looking at Figure 6, it is clear that the feature moved from left to right. The characteristics of the speedlines are similar to that of Figure 5. The line style is

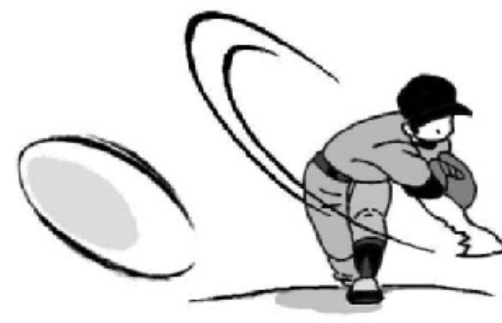

Figure 4: This illustration shows the use of speedlines to depict motion of the pitcher in a single frame. Illustration provided courtesy of Kunio Kondo [9].

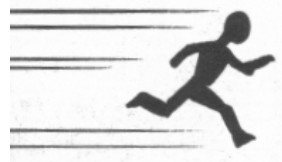

Figure 5: This illustration uses speedlines to depict the running motion of the man. The lines get thinner and lighter as they approach the running man. Illustration provided courtesy of HarperCollins publishers and Scott McCloud [14].

thicker and more opaque in older time instants and thinner, more translucent towards the newer time instants.

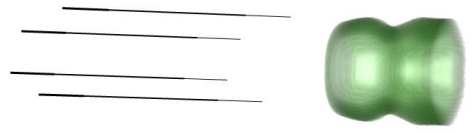

Figure 6: The image shows the use of speedlines to depict motion of the feature in the experimental dataset through twelve timesteps. The translatory motion of the feature from left to right is depicted using speedlines. The speedlines get thinner just and transclucent as they get closer to the latest timestep, just as Figure 5.

We applied the speedlines technique to actual CFD data to depict motion of a particular feature to get Figure 7. In this image, the motion of the feature is clearly depicted using speedlines. The downward motion of the feature is clearly conveyed to a viewer looking at this image.

Particle traces connect trajectories of a particle over time [5]. Particle traces are by definition required to be faithful to the path followed by the feature. A speedline, on the other hand, is an expression of how an illustrator would depict the same change. The speedlines approximate the path traced by the feature and incorporate the smooth, natural strokes of an illustrator to depict the motion of the feature. Speedlines differ from particle traces in that the line properties of speedlines are varied to depict the temporal change.

A streamline, by definition [5], is a line that is tangential to the instantaneous velocity direction and generally a collection of streamlines are used to convey flow. Speedlines, on the other hand, are used to track the motion of a feature over a certain interval of timesteps. The goal of using speedlines is not to convey flow for the entire time-varying dataset, but to facilitate tracking a feature of interest. Figure 8 illustrates the difference between using particle traces and speedlines. The leftmost image depicts a particle trace of the feature, the middle and rightmost image depict its motion us- 


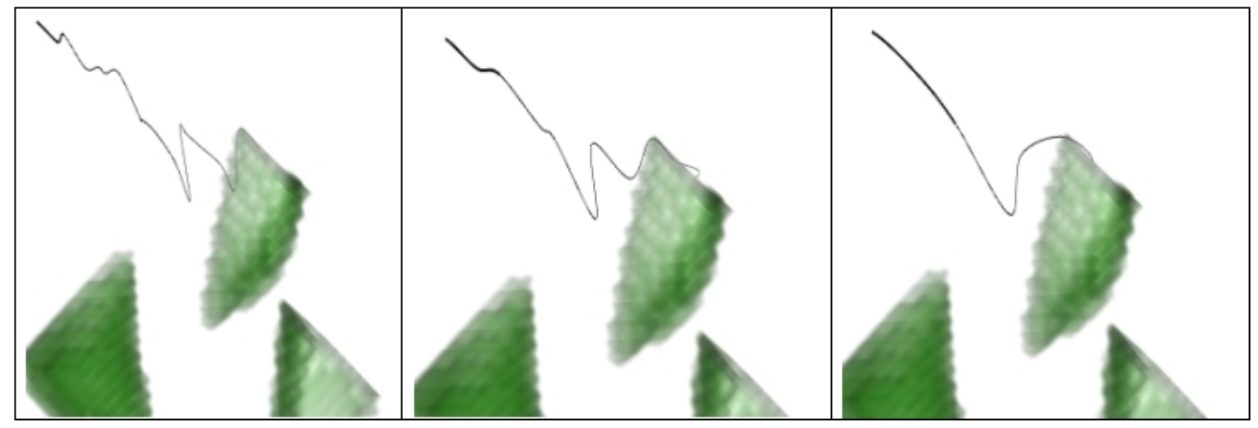

Figure 8: This set of images highlights the difference between particle traces and the speedlines technique. The leftmost image depicts a particle trace of the feature, the middle and rightmost image depict its motion using speedlines. In the middle image, we used alternate points from the path traced by the feature and in the rightmost image we use one out of four points to generate speedlines.

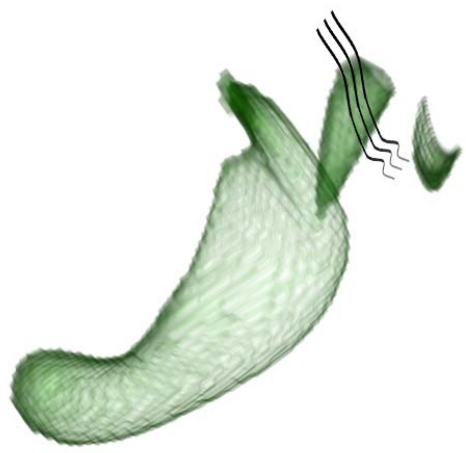

Figure 7: The image depicts change over time in a feature for CFD data. The downward motion of the flow feature is conveyed using speedlines.

ing speedlines. In the middle image, we used alternate points from the path traced by the feature and in the rightmost image we use one out of four points to generate speedlines. Our speedlines images are similar to the ones that illustrators would draw to convey the motion of the feature. The speedlines are smooth and convey the direction of motion to the viewer.

\subsection{Flow Ribbons}

Flow ribbons are used extensively by illustrators to show motion. Flow ribbons are particularly interesting because they occlude underlying regions to depict change. This facilitates the depiction of motion over time.

For example, in Figure 9, the illustrator has occluded parts of the monster's legs to depict the motion of his hand. At the same time, the small line segments, inside the flow ribbon (near his legs), serve as an abstraction to represent a simplified structure of the region of the legs occluded by the flow ribbon. Flow ribbons convey morphological change of the feature as well as the path of motion of the feature from the first time step to the current time step.

To obtain flow ribbons, we identify the centroid of a selected feature in every time step and we use that information to draw the flow ribbons. An important characteristic for flow ribbons is that they fade into the background and stop short of the feature. As can be seen in all the figures in Figure 10, this effect gives the viewer an opportunity to mentally complete the diagram by filling in the details. In this process of mentally completing the picture, the viewer is convinced of the change over time.

We define three different types of flow ribbons based on their

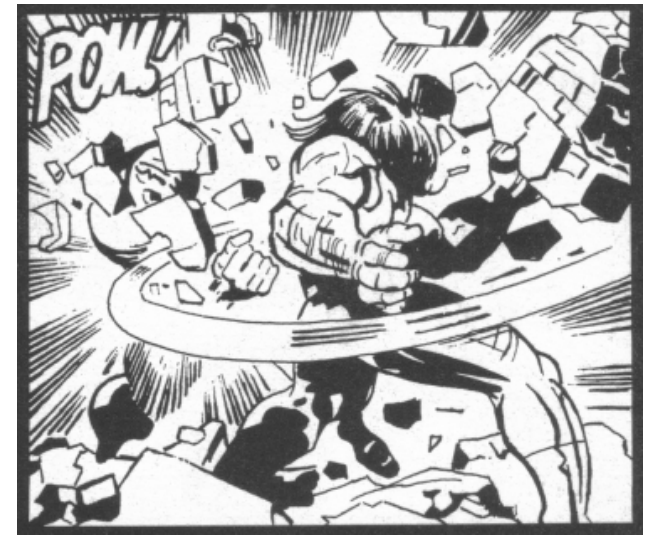

Figure 9: The ilustration depicts the motion of the monster's hand using a flow ribbon. The region near his thighs is occluded and is abstracted using small lines within the flow ribbon. The small lines convey to the viewer the presence of structure under the flow ribbon. Illustration provided courtesy of HarperCollins publishers and Scott McCloud [14].

complexity. The simplest flow ribbons are the ones in which a pair of speedlines are considered together to convey change over time. The second type of flow ribbons are opaque and occlude underlying features. It serves to draw the user's attention to the feature of interest. The third type of flow ribbons use small line segments to abstract the underlying occluded features. Examples for these three types of flow ribbons are shown in Figure 10.

To obtain the line segments overlapping underlying features, an alpha test followed by a stencil test is used. The alpha test checks for underlying features and the stencil test draws the line segments on the underlying feature to provide an abstraction for that feature. The line segments are dynamically generated as the volume is explored. In Figure 11, the helical motion of the experimental data feature is shown using flow ribbons. The flow ribbons depict a change of motion along the path of the ribbons. As per Figure 9, the regions where the flow ribbon occludes actual data, the line segments abstract their presence.

We applied the flow ribbons techniques to CFD data to track a feature in Figure 12. The ribbons occlude the underlying feature and abstract some part of it by small line segments that represent the feature, similar to Figure 9. The random motion from bottom left to upper right part of the figure is shown using flow ribbons.

Looking at Figure 12, the motion of the feature over time is conveyed. The motion of the feature with respect to the other features 


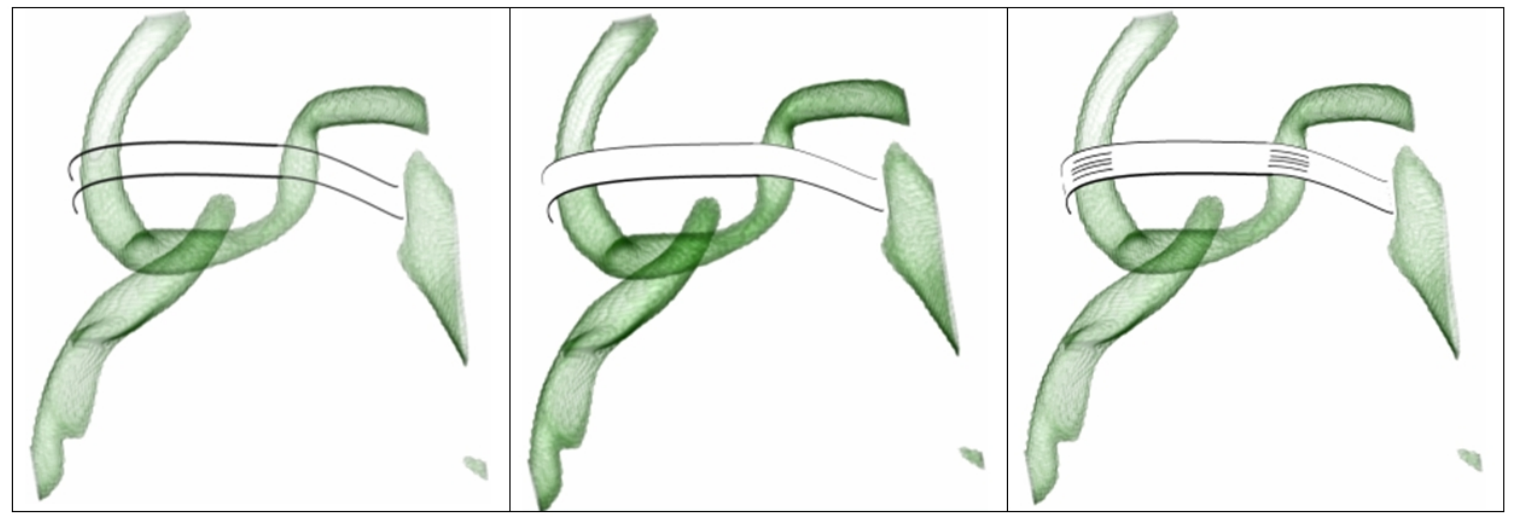

Figure 10: This set of images highlights the difference between various types of flow ribbons. In their simplest form, flow ribbons are merely pairs of speedlines that convey the change to the viewer. In the middle figure, the other extreme in which the region under the flow ribbon is occluded by the ribbon to draw the viewer's attention to the moving feature and emphasize its motion. In the rightmost figure, techniques similar to Figure 9 were used to generate the ribbons. Small line segments were used to abstract features occluded by the flow ribbons.

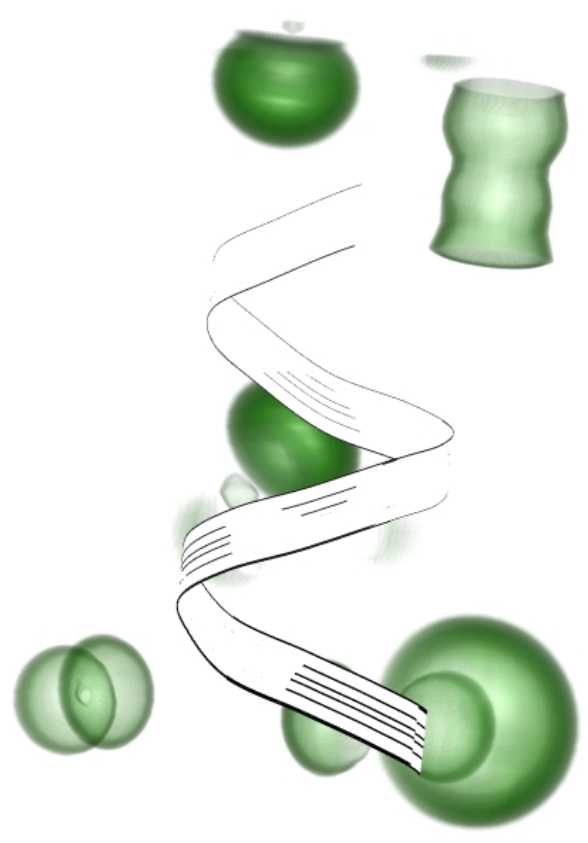

Figure 11: The image depicts the helical motion of the experimental data feature using flow ribbons. Just as in Figure 9, the occluded features are abstracted using small line segments. The flow ribbon gets thinner as they get closer to the newest timestep.

in the scene is clearly depicted.

\subsection{Opacity modulation}

Illustrators have often used techniques where they use a blurred, desaturated image to depict an older time step whereas a brighter, more detailed image represents a newer time step.

For this technique, we identify a particular interesting feature and then merge the rendering of the snapshots of each timestep into one image. At the same time, we modulate the opacity of the older timesteps and make them less opaque and dull whereas the newer timesteps are more opaque and the colors of the newer steps are brighter compared to the older time steps. This provides insight into

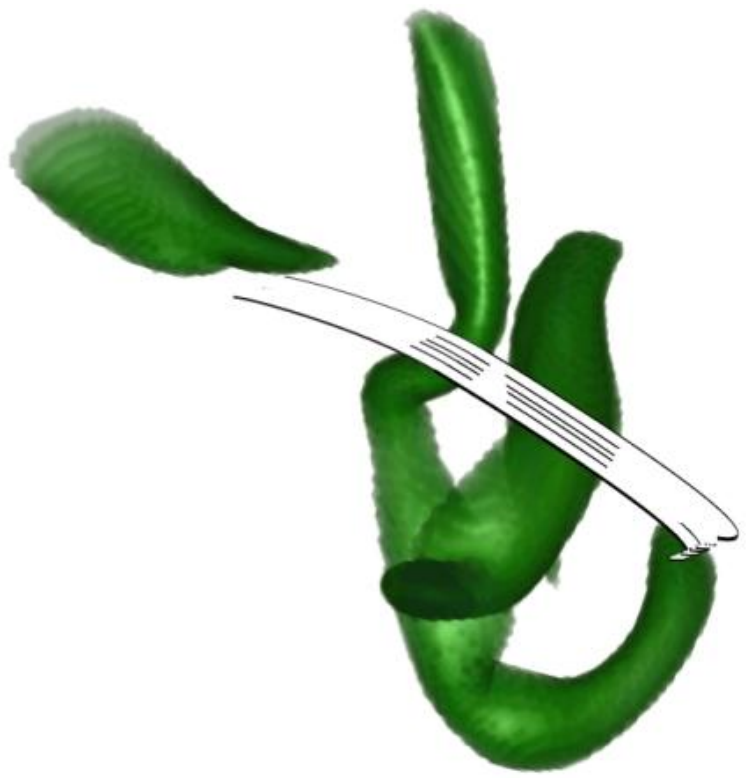

Figure 12: The image depicts random motion of a flow feature using flow ribbons. The feature moves from bottom right to upper left corner. The features occluded by the flow ribbon are abstracted using thin, small lines to represented the underlying features.

the origin of the feature and its path through multiple timesteps. We found that the opacity-based techniques in conjunction with speedlines were a better combination to convey the change over time than opacity-based techniques.

Figure 13 conveys the change over multiple timesteps to the viewer. The varying line thickness and increasing level of detail conveys the left-to-right motion of the hand.

We combined this technique with our speedlines technique to get Figure 14. The older time step is less saturated and dull, whereas the newer time step is brighter and more well defined compared to the blurred older time steps. This figure conveys the motion of the feature, from the left upper corner to the right bottom corner, to the viewer using a combination of the two techniques very effectively. Just as with the speedlines technique, the thickness of the older line decreases as the line gets closer to the newer timestep. 


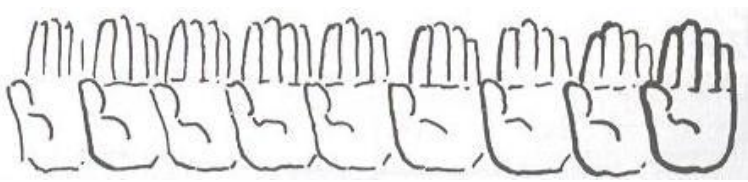

Figure 13: This illustration conveys the direction of motion of the hand over time. They use low detail, thin lines for the older instants of time and high detail, darker lines for the latest positions of the hand to convey the motion. Illustration provided courtesy of HarperCollins publishers and Scott McCloud [14].

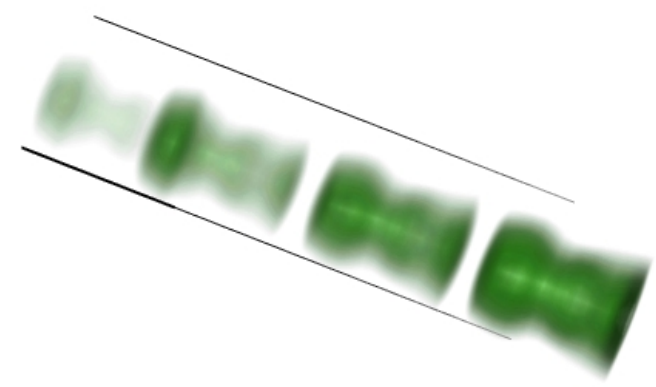

Figure 14: The image conveys change over time using a combination of the opacity-based technique with the speedlines based technique. The translatory motion of the feature from upper left to bottom right is conveyed by the image.

\subsection{Strobe silhouettes}

Illustrators have used strobe silhouettes to convey the previous positions of the object. As can be seen in Figure 15 the direction of motion of the axe is apparent from the trailing silhouette. The strobe silhouettes are increasing in their level of detail as they get closer to the current position of the object. The oldest time step has the most abstract, low level-of-detail silhouette. The trailing silhouette effect convincingly conveys the motion of the axe.

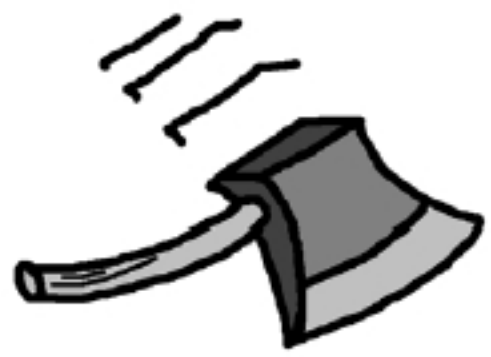

Figure 15: The image shows strobe silhouettes depicting motion over time. The downward motion of the axe is conveyed using strobe silhouettes. Illustration provided courtesy of Kunio Kondo [9].

To obtain strobe silhouettes, we precompute a direction-ofmotion vector for the feature. The dot product of the direction-ofmotion vector with the gradient vector gives us a trailing silhouette for every time step. We combine the silhouettes to get the strobe silhouette effect.

$$
\left(\nabla f_{n} \cdot \text { motionvector }\right)<0 \Longrightarrow \text { Strobesilhouette }
$$

Figure 16 for the experimental dataset uses strobe silhouettes to convey a translation in the horizontal direction to the viewer. The feature starts from the left extreme and translates to its current position.

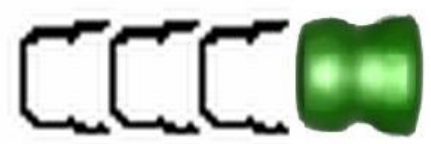

Figure 16: The strobe silhouettes technique applied to the experimental data set. The horizontal motion of the feature from left to right is depicted using the strobe silhouettes technique.

Figure 17 shows the upward motion of the flow features using strobe silhouettes. The direction-of-motion vector facilitates the generation of trailing silhouettes. The silhouettes are not as smooth as an illustrator would draw them and we are looking at line-based techniques for strobe silhouette generation [1].

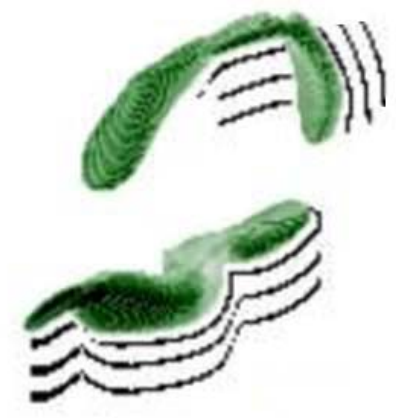

Figure 17: The strobe silhouettes technique applied to flow data. The strobe silhouettes convey the upward motion of the two features. The direction-of-motion vector enables the generation of trailing silhouettes.

Strobe silhouettes are extremely effective in conveying the direction of motion to the viewer because they provide an abstraction of past time steps. The viewer can mentally recreate the motion with the help of these strobe silhouettes and that helps in conveying change over time to the viewer.

\section{Discussion}

The suitability of these techniques is highly dependent on the type of motion the feature undergoes. The strobe silhouettes or the opacity-based techniques are not suitable for types of motion where the feature re-traces the path it has followed because the silhouettes will overlap each other making it harder for the viewer to track the feature and disambiguate older silhouettes with newer ones. Similarly, for opacity-based techniques an older timestep will be occluded by newer timesteps and can cause confusion for the viewer.

For such motion, the speed lines or the flow ribbons would be more suitable. Among other types of motion, the speedlines as well as the flow ribbons techniques would be suitable for twisting motion. Figure 11 is a great example that depicts spiral motion of the feature using flow ribbons.

We know that all the flow features are moving at all times. So far, we have shown the motion of a single feature over time. We have included some preliminary results in tracking multiple flow features over time. Figure 18 shows the motion of three features. Flow 
ribbons are used to show the motion of a feature and speedlines are used for the motion of the other two features. Since flow ribbons occlude underlying flow features, we use flow ribbons to depict the motion of higher priority features and speedlines are used for lower priority flow features. The central feature is moving leftwards and its motion is shown using a flow ribbon. The topmost feature's rightward motion and the bottommost feature's leftward motion is shown using speedlines.

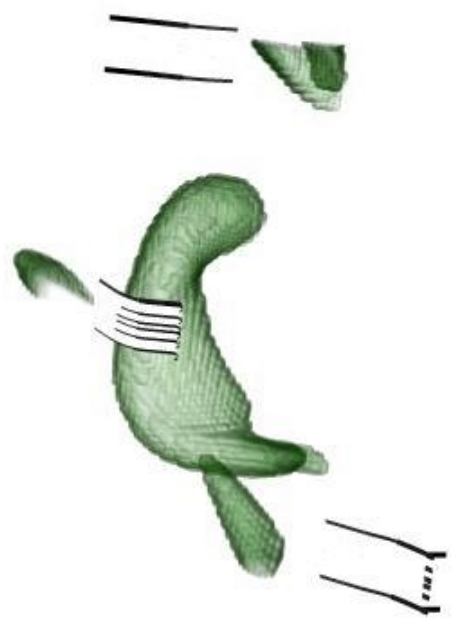

Figure 18: Multiple feature tracking using flow ribbons for the higher priority flow feature and speedlines for the lower priority flow features. The central feature's leftward motion is represented using a flow ribbon. The rightward motion of the topmost feature and the leftward motion of the bottommost feature is represented using speedlines.

This brings us to the use of multiple techniques in a single visualization. We have already seen the combination of speedlines and opacity-based techniques in Figure 14. Figure 19 is another example that uses flow ribbons and opacity-based technique to convey change over time. This is the actual motion of the feature whose snapshots are shown in Figure 2. It is evident that this single image (Figure 19) effectively conveys the rotational motion of the feature compared to the five snapshots in Figure 2. The combination of opacity-based techniques and flow ribbons concisely captures the feature's rotational motion.

\section{Conclusions}

Visualizing time-varying datasets is more challenging than threedimensional volume visualization. Each dataset is made up of multiple time steps and requires analysis to identify relevant features. To facilitate the analysis of the data, the visualization of data using illustration-inspired techniques is proposed. We have identified and substantiated our claim by examples proving that our techniques of using speedlines, flow ribbons, opacity-modulation and strobe silhouettes are effective in conveying change over time. We propose to conduct a user study to substantiate our claims and confirm the efficacy of our techniques. For the strobe silhouettes technique, we are investigating line-based techniques introduced by Burns et al. [1] to generate smoother silhouettes to more closely resemble illustrated silhouettes.

\section{ACKNOWLEDGEMENTS}

We would like to thank Dr. Deborah Silver and Kristina Santilli for providing the Vortex dataset and for many fruitful discussions. We

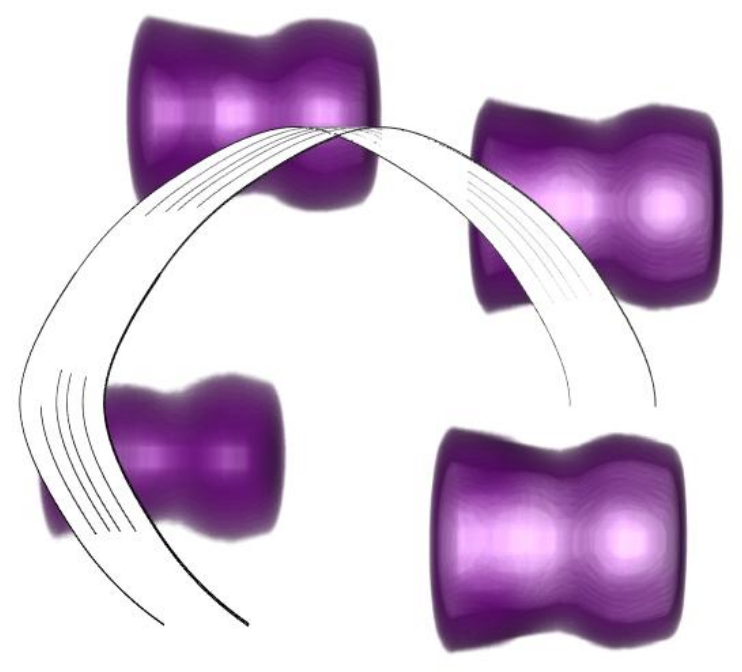

Figure 19: The rotational motion of the feature in the experimental dataset is conveyed using a combination of opacity-based techniques and flow ribbons. This figure conveys the actual motion of the feature whose snapshots are shown in Figure 2. This motion is not at all obvious by looking at the snapshots in Figure 2 .

thank Joe Kniss and Gordon Kindlmann for providing the Simian renderer. We would like to thank HarperCollins publishers, Scott McCloud and Kunio Kondo for permitting the use of their illustrations in the paper. We would like to thank the anonymous reviewers who raised valid points and helped immensely by their comments. This work has been funded by NSF grant numbers 0121288 and 0081581 .

\section{REFERENCES}

[1] Michael Burns, Janek Klawe, Szymon Rusinkiewicz, Adam Finkelstein, and Doug DeCarlo. Line drawings from volume data. ACM Transactions on Graphics (SIGGRAPH 2005), 24(3), August 2005.

[2] Brian Cabral, Nancy Cam, and Jim Foran. Accelerated volume rendering and tomographic reconstruction using texture mapping hardware. In Proceedings of the 1994 symposium on Volume visualization, pages 91-98. ACM Press, 1994.

[3] Balázs Csèbfalvi, Lukas Mroz, Helwig Hauser, Andreas König, and Eduard Gröller. Fast visualization of object contours by nonphotorealistic volume rendering. In Proceedings of the Eurographics Conference '01, volume 20(3), 2001.

[4] David Ellsworth, Ling-Jen Chiang, and Han-Wei Shen. Accelerating time-varying hardware volume rendering using tsp trees and colorbased error metrics. In VVS '00: Proceedings of the 2000 IEEE symposium on Volume visualization, pages 119-128. ACM Press, 2000.

[5] Richard S. Gallagher. Computer Visualization: Graphics Techniques for Scientific and Engineering Analysis. CRC Press, Inc., Boca Raton, FL, USA, 1994.

[6] Markus Hadwiger, Christoph Berger, and Helwig Hauser. Highquality two-level volume rendering of segmented data sets on consumer graphics hardware. In Proceedings of the conference on Visualization '03, pages 40-47. IEEE Computer Society, 2003.

[7] Elaine R. S. Hodges. The Guild Handbook of Scientific Illustration. John Wiley and Sons, 1989.

[8] T.J. Jankun-Kelly and Kwan-Liu Ma. A study of transfer function generation for time-varying volume data. In Proceedings of Volume Graphics, 2001, 2001.

[9] Yuya Kawagishi, Kazuhide Hatsuyama, and Kunio Kondo. Cartoon blur: Non-photorealistic motion blur. In Proceedings of the Computer 
Graphics International Conference, pages 276-281. IEEE Computer Society, 2003.

[10] Aidong Lu, Christopher J. Morris, David Ebert, Penny Rheingans, and Charles Hansen. Non-photorealistic volume rendering using stippling techniques. In Proceedings of the conference on Visualization '02, pages 211-218, 2002.

[11] Eric B. Lum and Kwan-Liu Ma. Hardware-accelerated parallel nonphotorealistic volume rendering. In Proceedings of the second international symposium on Non-photorealistic animation and rendering, pages 67-ff, 2002.

[12] Eric B. Lum, Kwan Liu Ma, and John Clyne. Texture hardware assisted rendering of time-varying volume data. In Proceedings of the conference on Visualization '01, pages 263-270. IEEE Computer Society, 2001.

[13] Edward MacCurdy. The Notebooks of Leonardo Da Vinci, volume 1. The Reprint Society London, 1954.

[14] Scott McCloud. Understanding Comics. HarperCollins publishers, 1994.

[15] Zenon W. Pylyshyn. Seeing and Visualizing: Its not what you think (Life and Mind). Bradford Book, 2003.

[16] Penny Rheingans and David Ebert. Volume illustration: Nonphotorealistic rendering of volume models. In IEEE Transactions on Visualization and Computer Graphics, volume 7(3), pages 253-264, 2001.

[17] Ravi Samtaney, Deborah Silver, Norman Zabusky, and Jim Cao. Visualizing features and tracking their evolution. Computer, 27(7):20-27, 1994.

[18] Han-Wei Shen, Ling-Jen Chiang, and Kwan-Liu Ma. A fast volume rendering algorithm for time-varying fields using a time-space partitioning (tsp) tree. In Proceedings of the conference on Visualization '99, pages 371-377. IEEE Computer Society Press, 1999.

[19] Han-Wei Shen and Christopher R. Johnson. Differential volume rendering: a fast volume visualization technique for flow animation. In VIS '94: Proceedings of the conference on Visualization '94, pages 180-187. IEEE Computer Society Press, 1994.

[20] Deborah Silver and X. Wang. Volume tracking. In Proceedings of the 7th conference on Visualization '96, pages 157-ff. IEEE Computer Society Press, 1996.

[21] Aleksander Stompel, Eric B. Lum, and Kwan-Liu Ma. Featureenhanced visualization of multidimensional, multivariate volume data using non-photorealistic rendering techniques. In Proceedings of $\mathrm{Pa}$ cific Graphics 2002 Conference, 2002.

[22] Philip Sutton and Charles D. Hansen. Isosurface extraction in timevarying fields using a temporal branch-on-need tree (t-bon). In Proceedings of the conference on Visualization '99, pages 147-153. IEEE Computer Society Press, 1999.

[23] Nikolai Svakhine, Yun Jang, David Ebert, and Kelly Gaither. Illustration and photography inspired visualization of flows and volumes. In Proceedings of IEEE Visualization 2005, 2005.

[24] Jane Wilhelms and Allen Van Gelder. Octrees for faster isosurface generation. In Proceedings of the 1990 workshop on Volume Visualization, pages 57-62. ACM Press, 1990. 